\title{
BMJ Open Nurse-led clinic for patients with liver cirrhosis - effects on health-related quality of life: study protocol of a pragmatic multicentre randomised controlled trial
}

\author{
Maria Hjorth, ${ }^{1,2}$ Daniel Sjöberg, ${ }^{1}$ Anncarin Svanberg, ${ }^{2,3,4}$ Elenor Kaminsky, ${ }^{3}$ \\ Sophie Langenskiöld, ${ }^{3}$ Fredrik Rorsman ${ }^{2}$
}

To cite: Hjorth M, Sjöberg D, Svanberg A, et al. Nurseled clinic for patients with liver cirrhosis-effects on health-related quality of life: study protocol of a pragmatic multicentre randomised controlled trial. BMJ Open 2018;8:e023064. doi:10.1136/ bmjopen-2018-023064

\section{- Prepublication history for} this paper is available online. To view these files, please visit the journal online (http://dx.doi org/10.1136/bmjopen-2018023064).

Received 19 March 2018 Revised 21 June 2018 Accepted 8 August 2018

A) Check for updates

(c) Author(s) (or their employer(s)) 2018. Re-use permitted under CC BY-NC. No commercial re-use. See rights and permissions. Published by BMJ.

${ }^{1}$ Center of Clinical Reaerch in Dalarna, Falun, Sweden

${ }^{2}$ Department of Medical

Sciences, Uppsala Universitet

Medicinska fakulteten, Uppsala, Sweden

${ }^{3}$ Department of Public Health and Caring Sciences, Uppsala University, Falun, Sweden

${ }^{4}$ Dalarna University, Falun, Sweden

Correspondence to Mrs Maria Hjorth; maria.hjorth@medsci.uu.se

\section{ABSTRACT}

Introduction Liver cirrhosis affects health-related quality of life (HRQoL) even in its early stages. Morbidity is especially high when the disease decompensates and self-care actions become essential. Nurse involvement in secondary prevention in other chronic diseases has contributed to better symptom control, less need of inpatient care and improved HRQoL. In order to evaluate the impact of nurse involvement in the follow-up of patients with liver cirrhosis, we decided to compare structured nurse-led clinics, inspired by Dorothea Orem's nursing theory and motivational strategies, with a group of patients receiving standard care. The primary outcome is $H R Q \mathrm{oL}$ and the secondary outcomes are quality of care, visits to outpatient clinics or hospitals, disease progress and health literacy.

Methods and analysis This is a pragmatic, multicentre randomised controlled study conducted at six Swedish hepatology departments. Eligible patients are adults with diagnosed cirrhosis of the liver $(n=500)$. Participants are randomised into either an intervention with nurseled follow-up group or into a standard of care group. Recruitment started in November 2016 and is expected to proceed until 2020. Primary outcomes are physical and mental HRQOL measured by RAND-36 at enrolment, after 1 and 2 years.

Ethics and dissemination The study is ethically approved by the Regional Ethical Review Board in Uppsala. The results shall be disseminated in international conferences and peer-reviewed articles.

Trial registration number NCT02957253; Pre-results.

\section{INTRODUCTION}

The incidence of liver cirrhosis in Sweden is approximately 14 per 100000 citizens each year. ${ }^{1}$ It is a disease with high mortality as well as high morbidity, affecting patient's health-related quality of life (HRQoL). Fatigue and depression are already frequent during the early, compensated, phase of liver cirrhosis and are believed to impair HRQoL by affecting the patient's social life. ${ }^{2}$ HRQoL
Strengths and limitations of this study

- This pragmatic multicentre randomised controlled study design enables evaluation of a nurse-led clinic intervention in patients with liver cirrhosis in the real-life context.

- All nurses involved in the study are proficient in the field of liver diseases, having a holistic understanding of the situation of liver cirrhosis.

- The generic health-related quality of life instrument RAND-36 is used as a Swedish version of a liver-specific instrument is currently unavailable.

- There is a risk of unwittingly transferring the intervention to the control group. This is counteracted by the multicentre design and will shorten the time for recruitment of participants.

is further impaired in the decompensated patients, when symptoms of ascites, hepatic encephalopathy (HE) or variceal haemorrhage occur. ${ }^{34}$

In the compensated stages, lifestyle changes are important to prevent or delay disease progression. While in the decompensated phase, customised lifestyle changes and selfcare become essential in the management of the disease. ${ }^{5}$ Unstructured follow-up in outpatient settings causes frequent readmissions due to the reappearance of complications of cirrhosis. The reason may be drug-related side effects, for example, diuretics, non-adherence to self-care or medical treatment. One-third of these episodes are said to be preventable with closer follow-up in an outpatient setting. ${ }^{67}$

Motivating patients for self-care activities is essential in nursing care. For this, Orem's theory of nursing, ${ }^{8}$ consisting of the three theories: self-care, self-care deficit and the nursing system may be applied. This theory 
guides nurses to identify and support patients to enter selfcare for better symptom control and improved health. ${ }^{8-10}$ In liver cirrhosis, management of patients with liver cirrhosis is traditionally taken care of by physicians, while nurse-led clinics are still rare. However, previous studies on liver cirrhosis, nurse-led clinics have suggested that nurse-led clinics will contribute to better patient concordance with physician recommendations ${ }^{11-13}$ and medical treatment, ${ }^{11} 12$ with positive effects on patients HRQoL. ${ }^{12}$ Furthermore, there are indications that nurse-led clinics increase the quality of care by increasing the number of patients treated according to medical healthcare guidelines. ${ }^{12}$ Finally, the patients have reported a high degree of satisfaction by nursing care in such outpatient settings. ${ }^{12} 14$ Despite these reports, the significance of adjunctive nurse-led clinic to standard care by physician in liver cirrhosis is unclear. Conversely, in chronic heart failure, nurse-led care is established and proved equally effective as traditional care by the physician within outpatient settings. ${ }^{15}$ The holistic and person-centred approaches by the nurse, including motivational strategies, have been shown to be crucial in the secondary prevention of chronic heart disease, reducing the need for inpatient care and to increase HRQoL. ${ }^{16}{ }^{17}$ Hence, the experience from nurse-led clinics in chronic heart disease is likely to provide guidance regarding content, methods and necessary skills in the set-up of nurse-led clinics within the field of liver cirrhosis.

The aim with the present study is to compare HRQoL in patients with liver cirrhosis receiving either adjunctive nursing care based on Orem's nursing theory or standard care only in outpatient settings.

\section{METHODS AND ANALYSIS}

The protocol follows the statement of Standard Protocol Items: Recommendations for Interventional Trials 2013, ${ }^{18}$ for study protocol and Template for Intervention Dscription and Replication (TIDieR). ${ }^{19}$.

\section{Study design}

The study has a pragmatic, multicentre randomised controlled comparative design.

\section{Study arms}

Patients in the intervention group obtain structured visits to nurse-led clinics depending on the severity of the disease. The intervention is adjunctive, that is, the intervention is added to standard care. Patients in the control group get standard inpatient and outpatient care according to clinical routines.

\section{Study sites}

The study settings consist of six outpatient clinics at hepatology departments in Sweden, two county hospitals and four university hospitals. None of the clinics had structured nursing care for patients with liver cirrhosis at the beginning of the study. The six outpatient clinics serve

\begin{tabular}{|c|c|}
\hline Inclusion criteria & Exclusion criteria \\
\hline $\begin{array}{l}\text { Diagnosed liver } \\
\text { cirrhosis within the past } \\
24 \text { months }\end{array}$ & $\begin{array}{l}\text { Insufficient knowledge of the } \\
\text { Swedish language }\end{array}$ \\
\hline $\begin{array}{l}\text { Follow-up at the } \\
\text { hepatology department }\end{array}$ & $\begin{array}{l}\text { Persistent hepatic encephalopathy } \\
\text { grades } 2-4\end{array}$ \\
\hline \multirow[t]{8}{*}{ Age $18-85$ years } & Comorbidity \\
\hline & $\begin{array}{l}\text { Chronic obstructive pulmonary } \\
\text { disease grades } 3-4\end{array}$ \\
\hline & $\begin{array}{l}\text { Coronary heart disease New York } \\
\text { Heart Association Functional } \\
\text { Classification (NYHA) classes 3-4 }\end{array}$ \\
\hline & Dementia \\
\hline & Actual advanced cancer \\
\hline & Stroke with sequelae \\
\hline & Severe psychiatric disease \\
\hline & Renal failure requiring dialysis \\
\hline
\end{tabular}

a population of approximately 2000000 individuals, comprising about $20 \%$ of Sweden's population.

\section{Eligibility criteria}

Diagnosis of liver cirrhosis is based on clinical investigation, laboratory findings, histology, MRI, computer tomography, ultrasound or elastography. Factors likely to strongly affect the primary variable due to other reasons than liver cirrhosis, that is, severe comorbidities and those unable to adhere to the study protocol, that is, persistent, overt HE, are excluded. Inclusion and exclusion criteria are presented in table 1.

\section{Screening and recruitment of participants}

Invitation letters are sent by intervention nurses (INs), offering oral information. Patients are invited to a screening visit to IN for baseline measurements. Those who meet inclusion criteria are registered. Patients, who agree to participate, hereafter denoted as participants, are randomised after giving informed consent. Newly diagnosed patients are recruited consecutively (figure 1). INs are responsible facilitators and consecutively follow participants.

\section{Randomisation}

Computerised randomisation (Randomize.Net, Interrand, Ottawa, Canada) is performed at the screening visit with randomly mixed block sizes of 4, 6 and 8, stratified by study site and disease severity in terms of compensated or decompensated state (figure 1). Blinding of the randomisation sequence is applicable to all involved personnel; allocation will be 1:1. Baseline measurements are completed before randomisation. Further blinding is not possible in this study. 


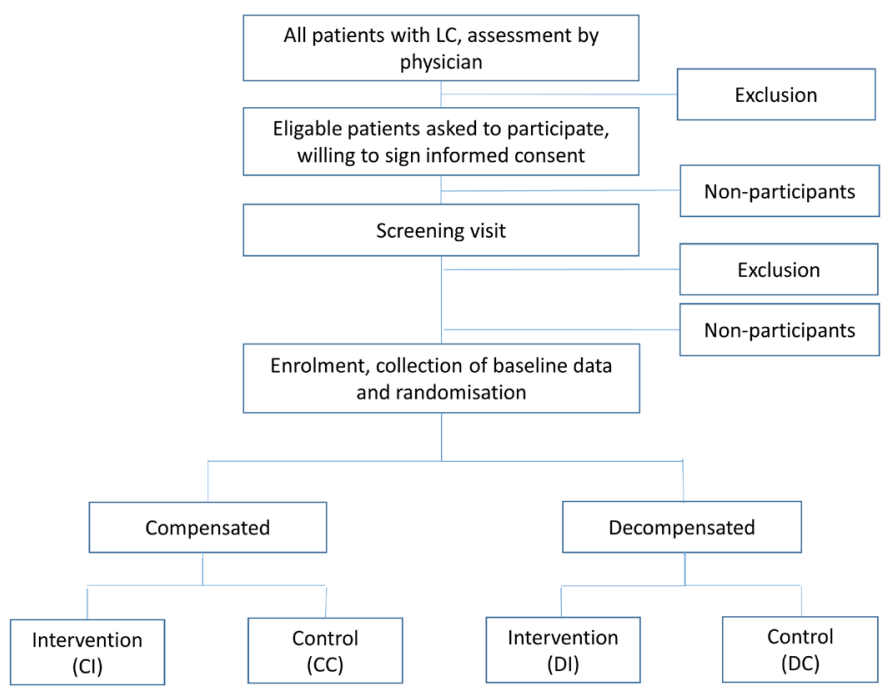

Figure 1 Recruitment and randomisation of participants. CC, compensated control group; Cl, compensated intervention group; DC, decompensated control group; DI, decompensated intervention group; LC, liver cirrhosis.

\section{Description of the intervention}

Participants in the intervention group offer scheduled individual visits to INs at the nurse-led clinic, in addition to visits to a physician according to clinical practice. Intervals between visits to the nurse-led clinic are varying from once yearly in compensated stable disease, up to two visit per month in decompensated disease (figure 2). The tailored frequency and content of visits are individualised to promote person-centred care (table 2).

Participants in the control group will receive standard care by physicians within hepatology inpatient or outpatient clinics as required and a yearly follow-up for data collection by IN within the study (figure 2).

\begin{tabular}{|c|c|c|c|c|}
\hline Baseline measurements: & & 12 month measurements: & & 24 month measurements: \\
\hline Background variables * & & RAND-36 & & RAND-36 \\
\hline RAND-36 & & QPP & & QPP \\
\hline QPP & & Review of health care consumption & & Review of health care consumption \\
\hline Symptoms of decompensation & Visits to $\mathrm{IN}^{* *}$ & Symptoms of decompensation & Visits to $\mathbf{I N}^{* *}$ & Symptoms of decompensation \\
\hline Child Pugh score & Or & Child Pugh score & Or & Child Pugh score \\
\hline MELD score & Standard & MELD score & Standard & MELD score \\
\hline RFH-NPT score & care*** & RFH-NPT score & Care*** & RFH-NPT score \\
\hline PHES-test & & PHES-test & & PHES-test \\
\hline CRT & & CRT & & CRT \\
\hline NVS & & NVS & & NVS \\
\hline
\end{tabular}

\footnotetext{
* gender, age at diagnosis, time since diagnosis, education level, accommodation, country of birth, employment and comorbidity

** Visits to IN according to disease severity

Compensated disease: Once yearly

Decompensated disease within 12 months: 1-2 visits/month

Previously decompensated disease: every 3rd month

Visits may alternately consist of telephone follow-up

*** Control group: Standard of care to physician as required
}

Figure 2 Study measurements and intervention nurse visit interval. CRT, continuous reaction time; IN, intervention nurse; MELD, Model for End-Stage Liver Disease; NVS, Newest Vital Sign; PHES-test, psychometric HE score; QPP, quality of care from the patient's perspective; RFH-NPT, Royal Free Hospital-Nutritional Prioritising Tool. 
Table 2 Description of the intervention

\begin{tabular}{|c|c|c|}
\hline Disease severity & Frequency of visits & Content of visits \\
\hline Compensated disease & Once yearly & $\begin{array}{l}\text { Child Pugh score } \\
\text { Model for End-Sage Liver Disease (MELD)-score } \\
\text { The Royal Free Hospital-Nutritional Prioritising Tool } \\
\text { Assessment of: ascites, encephalopathy } \\
\text { Motivation to lifestyle changes } \\
\text { Psychosocial issues }\end{array}$ \\
\hline $\begin{array}{l}\text { Decompensated disease } \\
\text { within } 12 \text { months or }\end{array}$ & $1-2$ visits/month & $\begin{array}{l}\text { Child Pugh score } \\
\text { MELD score }\end{array}$ \\
\hline $\begin{array}{l}\text { Previously decompensated } \\
\text { disease }\end{array}$ & Every third month & $\begin{array}{l}\text { The Royal Free Hospital-Nutritional Prioritising Tool } \\
\text { Assessment of: ascites, encephalopathy, side effects of medical treatment } \\
\text { Motivation to self-care and/or lifestyle changes } \\
\text { Psychosocial issues }\end{array}$ \\
\hline
\end{tabular}

Each visit to INs contains assessment of disease severity to enable early action against disease progression and malnutrition (table 2). The intervention includes treatment and nursing care inspired by Dorothea Orem's nursing theory. ${ }^{10}$ Further, motivational interviewing (MI) ${ }^{20}$ communication strategies will be used. Both Orem's theory and MI implies that individuals have an intrinsic motivation to make appropriate choices, to promote health and prevent disease or to perform actions to counteract disease. ${ }^{1020}$ The task of the IN is to assess the participants' self-care needs and their ability to perform essential self-care in order to discover self-care deficits. To evoke participants' motivation, INs listen and reflect on preparatory and mobilising change talk. In addition, INs give information adherent to MI techniques to facilitate participants understanding of actual self-care and medical treatment (figure 3). When applicable, INs offer next of kin instructions to help the participant achieve self-care.
The areas of the intervention are: (1) monitoring risk factors for deterioration of the liver disease, (2) information and motivation to perform self-care and adhere to medical treatment, (3) nutrition assessment and support, (4) motivation of lifestyle changes essential for preventing or delaying disease progress and (5) psychosocial care. A booklet written by MH is handed to all INs describing these five areas converted into terms of Orem's nursing theory.

One objective of INs' use of MI is to promote engagement and increased collaboration between IN and participant via the MI spirit concepts: partnership, evocation, compassion and acceptance. Another objective is that INs evoke participants' own motivation and explore patients' own thoughts about a target behaviour when there is need for behavioural change. When participants express mobilising 'change talk, ${ }^{20}$ they are ready for the planning phase (figure 3). The intervention is individually tailored and INs' activities depend on actual needs. An

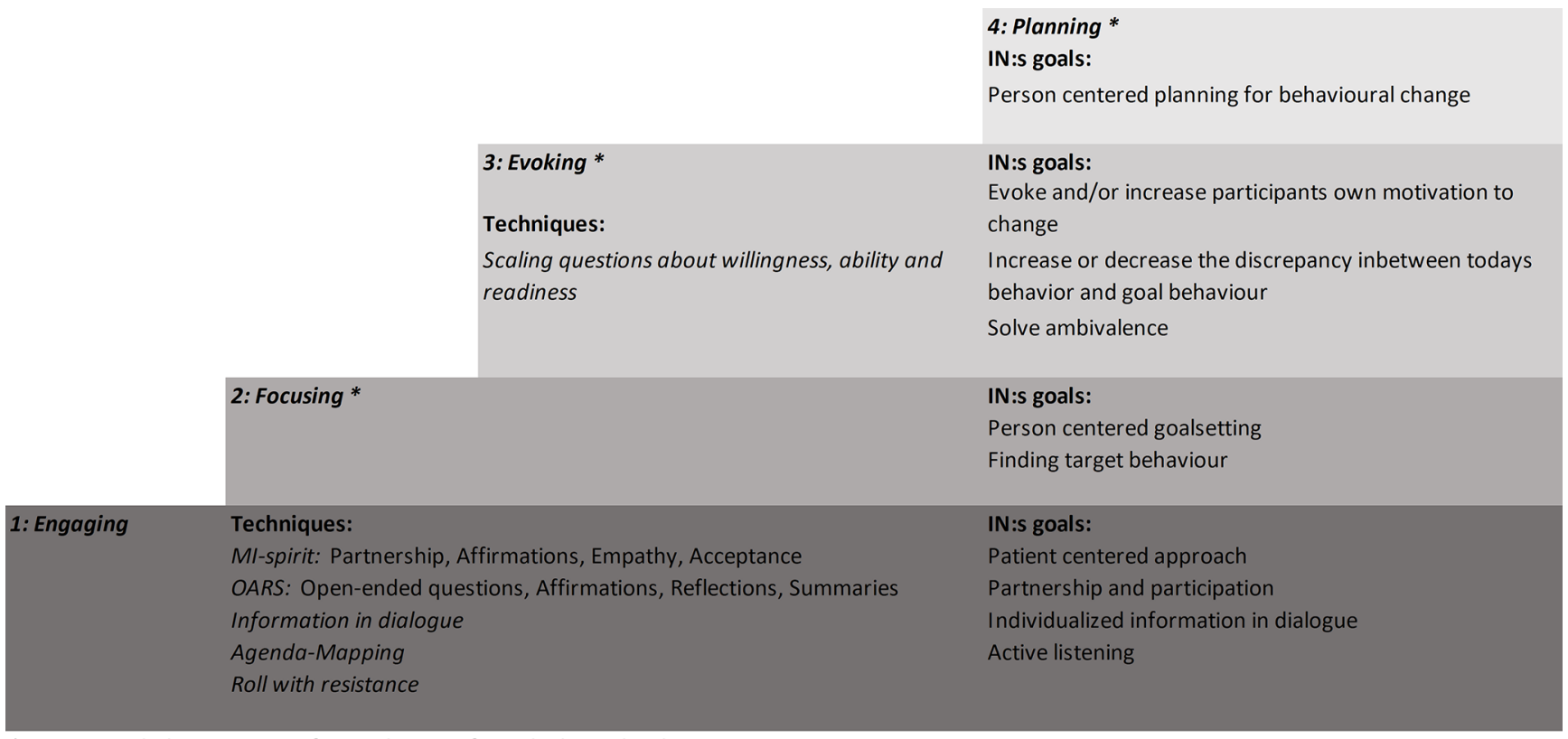

* Step 2-4 include a variation of MI techniques from the lower levels

Figure 3 The four processes of motivational interviewing techniques. IN, intervention nurse; MI, motivational interviewing. 
information booklet about liver cirrhosis is available to participants as a complement to oral information.

Standard care includes flexible visits or telephone follow-up by physicians, gastroscopies, ascites drainage, registered nurse telephone counselling by a nurse not participating in the study and inpatient care.

\section{Intervention nurses}

At each of the six clinics, 1-2 INs are involved in the intervention. All of these are registered nurses with a minimum of 2 years experience from hepatology inpatient or outpatient care. Implementation of the intervention and training of INs include a 6-hour seminar with a short description of MI and Orem theories followed by a 3-day training to perform MI. INs are also educated in pathophysiology of liver cirrhosis, nursing care according to presenting symptoms, study bias and study instruments. Scheduled tutorial group sessions to follow the intervention and MI practice will be due every 6 months for all INs during the study period.

\section{Study piloting}

A pilot of the intervention and patient questionnaire was performed in Falun from 2014 to 2015 with 26 participating patients. The aim was to define the actual size of the population available for the study and to assess the time and budget for the INs' assignment.

\section{Baseline sociodemographic data collection}

Sociodemographic data collected at enrolment are presented in figure 2.

\section{Primary outcome}

Physical and mental HRQoL are the two main outcomes in the present study measured by RAND-36. ${ }^{21}$ RAND-36 consists of 36 category scale questions: the answer to each question ranges from 0 to 100 , a higher value predicts better health. From the RAND-36 questionnaire, eight subscales are derived: (1) physical functioning, (2) role limitations caused by physical health problems, (3) pain, (4) energy/fatigue, (5) social functioning, (6) role limitations caused by emotional problems, (7) emotional wellbeing and (8) general health perception. Out of the eight subscales, two summary components are derived: Physical Component Summary (PCS) and Mental Component Summary (MCS). ${ }^{21}{ }^{22}$ HRQoL measurements by the RAND-36 has high validity and reliability to identify differences in HRQoL over time within and compared with patient populations with different chronic diseases. ${ }^{22}$

\section{Secondary outcomes}

- Patient's perspective of quality of care due to a change in follow-up strategy: The questionnaire quality of care from the patient's perspective (QPP) ${ }^{23}$ includes four dimensions: (1) medical-technical competence, (2) physical-technical conditions, (3) identity-orientated approach and (4) sociocultural atmosphere. Within each dimension, the participants first value their experience of the specific care aspects they have received
((1) totally agree, (2) agree in large part, (3) partly agree or (4) do not agree) and second, the importance of these aspects ((1) of greatest importance, (2) of great importance, (3) of some importance or (4) of little or no importance). The difference between the experienced care and the importance of each question is categorised as: excess of, balanced or lack of quality of care. A short form of QOP has been found valid and reliable. ${ }^{24}$ In the present study, participants receive a modified QPP 38-item questionnaire adjusted for patients with liver cirrhosis in outpatient care. The modification has been approved by the instrument developer. The questionnaire includes a variation of yes/no questions, category scales from 1 to 4 and open-ended questions.

- Visits at outpatient clinics and admissions to hospital: Visits at outpatient clinics, number of admissions to hospitals and days of inpatient care at medical wards or intensive care units will be recorded as measures of healthcare consumption. In case of significant clinical outcomes, these data will later be used to perform a separate health economic analysis.

\section{Disease progress}

1. Child Pugh score ${ }^{25}$ includes five variables: serum albumin, serum bilirubin, prothrombin time, ascites and encephalopathy. Each variable grading from 1 to 3 and the total range is $5-15$. A higher value means a more advanced disease. Three risk classes are derived: $\mathrm{A}=$ score $5-6, \mathrm{~B}=$ score $7-9$ and $\mathrm{C}=$ score $10-15{ }^{26}$

2. The Model for End-Stage Liver Disease (MELD) ${ }^{27}$ predicts the 3-month mortality of patients with chronic end-stage liver disease. Based on laboratory findings, MELD is a valid and reliable instrument. The formula for MELD is constant for disease aetiology, the calculation score is: $9.57 \mathrm{x} \log$ e (creatinine $\mathrm{mg} / \mathrm{dL})+3.78 \times \log$ e (bilirubin $\mathrm{mg}$ / $\mathrm{dL})+11.20 \times \log$ e (INR) +6.4 . The score is continuous, ranging from 6 to 40, a high score predicts an increased risk of mortality within 3 months. ${ }^{28}$

3. The Royal Free Hospital-Nutritional Prioritising Tool (RFH-NPT) ${ }^{29}$ assesses the risk of malnutrition in liver cirrhosis as a predictor of disease deterioration and transplant-free survival. RFH-NPT correlates with deterioration of the liver disease and divides participants into low (0 points), medium (1 point) or high (2-7 points) risk groups for malnutrition. Parameters taken into account are nutritional history (unplanned weight loss, dietary intake body mass index) and current complications of liver cirrhosis (acute alcoholic hepatitis, ascites, general fluid overload). The instrument used in the study is a translation into Swedish from the English version. Validation of the translation is made in a research seminar within the research group.

4. Appearance of decompensation episodes (eg, ascites, overt HE and variceal bleed) is assessed at screening, after 12 months and after 24 months 
through medical records. HE is common in liver cirrhosis with a cumulative risk of $30 \%-40 \%{ }^{30}$ According to the West-Haven criteria, ${ }^{31} \mathrm{HE}$ ranges from 0 to 4 . Grades $0-1$ mean subclinical or minimal symptoms (covert HE) and grades 2-4 mean severe neuropsychiatric symptoms (overt HE) ${ }^{30}$ Even milder grades of HE affect HRQoL. ${ }^{30}{ }^{31}$ In the majority of cases, HE is treatable. Two psychometric tests in combination are recommended to detect covert HE. ${ }^{30}$ In this study, the psychometric HE score (PHES) and continuous reaction time (CRT) are used:

a. PHES consists of five-step paper and pencil tests, ${ }^{32}$ and includes a line drawing test, a serial dotting test and a digit symbol test to examine motor speed and accuracy, visual perception, visuospatial orientation, visual construction, concentration and attention. The test ends up with a score ranging from +6 to $-18 ;-4$ or less is the cut-off for a pathological result.

b. $\mathrm{CRT}^{33}$ is a $10 \mathrm{~min}$ test with auditory stimuli in headphones in intervals of every 2-6s. It tests the reaction time and endurance by pushing a trigger button after a signal. Using the software EKHO reaction-time analysis tool, an index $<1$, 9 with 150 repetitions separate HE from other brain dysfunctions with a specificity of 0.92 and sensitivity of 0.93 .

- Health literacy (HL): involves a person's ability to receive, process and understand basic medical information in making decisions and taking actions to promote health. ${ }^{34}$ The grade of HL may influence the intervention as it impacts the participants' ability to understand and translate information into practice. The instrument Newest Vital Sign,${ }^{35}$ which consists of six standardised questions about nutrition label information, is used. The questions is asked by INs, the correct answer scores 1 point, a score of 4 or above indicates no limits of HL and scores below 4 indicate limited HL. The instrument is translated from English to Swedish within another study (Health literacy among Swedish lung transplant recipients 1-5 years after transplantation, A Lennerling, A Kisch and A Forsberg, personal communication, 2018). Validation of the translation has been made in a research seminar within our research group and the risk of translation errors was judged to be low.

\section{Participant flow through the study}

Study time for each participant is 24 months (figure 2), after which participants in the intervention group may continue their follow-up at the nurse-led clinic if they want to. Participants in the control group may be offered follow-up at the nurse-led clinic after the end of study. Data collection and frequency of visits to INs are presented in figure 2. Reasons for withdrawal from the study are liver transplantation, move out of follow-up area or mortality. In case of two consecutive cancelled visits to an IN, a reminder will be sent, asking participants to contact INs for further participation in the study.

\section{Data analysis and sample size calculation}

Analysis includes the two primary variables in RAND-36: the PCS and the MCS. These components will be calculated based on weights from the oblique method, ${ }^{36}$ to avoid potential problems in interpretation due to negative weights. A repeated measurements model will be used for analysis of baseline, 12 and 24 months values of the component summary score. Treatment group, time (baseline, 12, 24 months) with interaction, and decompensated/compensated state will be fixed effects, while site will be a random effect in the model. The main contrast of interest to be estimated with this model is change from baseline to month 24 for both treatment groups. For the treatment group comparison, Bonferroni-Holm method using a corrected alpha $=2.5 \%$ will be used to compensate for multiple testing of both PCS and MCS.

All tests will be two sided. For analyses of secondary variables, $p$ values $<0.05$ are considered significant. Multiple imputation will be used for missing values.

For the power calculation, a SD of 9 points was used based on back-calculated residual variances from CIs for MCS. ${ }^{37}$ A change of 3-5 points is estimated to be a minimally clinically important difference and corresponds to an effect size of $0.09-0.28 .^{22} \mathrm{It}$ is argued that even smaller changes are important, ${ }^{38}$ and a change of 2.5 points is therefore considered to be potentially relevant for the power calculation. To ensure a power of 0.80 for the effect size of 2.5 points and a Bonferroni-corrected alpha level of $2.5 \%$, the recommended sample size is 250 participants per treatment group, that is, 500 in total. ${ }^{39}$ With a calculated $33 \%$ non-inclusion rate, enrolment time is estimated from November 2016 to December 2020 or until 500 participants are included.

\section{Patient involvement}

Five patients contributed with comments on the questionnaire that resulted in changes in tree of the 78 questions. No patients were involved in study design, research question or recruitment. The results will be disseminated to the study participants in a short summary after the publication of the study.

\section{Strengths and limitations}

This randomised controlled trial in the field of nursing is a complex intervention with a pragmatic design and has a high risk of confounding factors. In a pragmatic design, ${ }^{40}$ the research aim is to reflect the clinical practice. Participant heterogeneity and a minimum of exclusion criteria are therefore allowed to a larger extent. The researcher must be aware of factors that may bias study results. In this study, participants may have other chronic diseases that may affect HRQoL. Patients with severe comorbidity are not included in this trial as it would have required a larger sample than available. A prolonged study of 5 years raises the risk of unwitting transfer of the intervention on to 
the control group when other personnel than INs start to develop skills included in the study protocol. ${ }^{17}$ To reduce this risk and preserve the ability to identify changes in HRQoL, study time is set to 4 years. Furthermore, six study sites are included in the study that aims to shorten the time for the study as well as reduce time for contamination of the control group. Six study sites, however, imply a risk of performance differences regarding the intervention and control group. In a forthcoming study, the context and mechanisms that may affect the results will be assessed in a process evaluation as described by Moore et $a l^{41}$ To reduce bias in inclusion, participants in the control group offer a nurse-led clinic when study participation ends.

Although RAND-36 has proved sensitivity to changes in liver cirrhosis, a disease-specific instrument may be more nuanced. However, available disease-specific HRQoL instruments for liver cirrhosis are unfortunately not translated into Swedish. The former comparable generic instrument SF-36 ${ }^{22}$ has been shown to be sensible for differences in HRQoL in populations of liver cirrhosis,. ${ }^{44}$

The MI experience may affect INs' ability to use MI skills in conversations with participants. However, all INs in the present study are proficient or experts in nursing in the field of liver cirrhosis, having a holistic understanding of the situation of the disease. During the study, the INs will attend tutorial sessions twice a year to develop their skills in MI.

The occurrence of overt HE limits the enrolment of participants. Overt HE may have an impact on the ability to answer questionnaires used in the study. At enrolment, patients with covert HE are accepted. Before measurements at 12 and 24 months, HE tests will be repeated before the participants answer the questionnaires. Participants with overt $\mathrm{HE}$ will not be asked to answer the questionnaires, including RAND-36 and QPP, due to the risk of unreliable answers.

To our knowledge, this is the largest randomised controlled study evaluating nurse-led intervention in liver cirrhosis. The pragmatic design enables us to evaluate the effect of the intervention in the real-life context under which the study is performed. The results will hopefully contribute with important knowledge about nurse involvement in the care of patients with liver cirrhosis that can be applied in the routine clinical setting.

\section{Dissemination}

The study result will be published in peer-reviewed journals and presented at international conferences. The result will be used for education and competence development within the field. Study results are reported on the group level.

Acknowledgements To Frank Miller for statistical support and to patient advisers for comments in development of the patient questionnaire.

Contributors MH has contributed to the design, implementation of the study and responsible for drafting the manuscript. DS has taken part in the design of the study and supervised MH in drafting the Manuscript, and has approved the final manuscript. AS has taken part in the design of the study and supervised $\mathrm{MH}$ in drafting the manuscript, and has approved the final manuscript. EK has taken part in the design of the study and supervised $\mathrm{MH}$ in drafting the Manuscript, and has approved the final manuscript. SL has taken part in the design of the study and supervised $\mathrm{MH}$ in drafting the manuscript, and has approved the final manuscript. FR has taken part in the design of the study and supervised $\mathrm{MH}$ in drafting the manuscript, and has approved the final manuscript.

Funding This work was supported by Ester Åsberg Lindberg foundation and Centre for Clinical Research in Dalarna. The CRT equipment was funded by Norgine.

Competing interests None declared.

Patient consent Obtained.

Ethics approval The study has been approved by the Regional Ethical Review Board in Uppsala (Dnr: 2016/146) and is performed according to the Declaration of Helsinki and to the Swedish Ethical Review Act.

Provenance and peer review Not commissioned; externally peer reviewed.

Open access This is an open access article distributed in accordance with the Creative Commons Attribution Non Commercial (CC BY-NC 4.0) license, which permits others to distribute, remix, adapt, build upon this work non-commercially, and license their derivative works on different terms, provided the original work is properly cited, appropriate credit is given, any changes made indicated, and the use is non-commercial. See: http://creativecommons.org/licenses/by-nc/4.0/.

\section{REFERENCES}

1. Nilsson E, Anderson H, Sargenti K, et al. Incidence, clinical presentation and mortality of liver cirrhosis in Southern Sweden: a 10-year population-based study. Aliment Pharmacol Ther 2016;43:1330-9.

2. Nardelli S, Pentassuglio I, Pasquale C, et al. Depression, anxiety and alexithymia symptoms are major determinants of health related quality of life (HRQoL) in cirrhotic patients. Metab Brain Dis 2013;28:239-43.

3. Arguedas MR, DeLawrence TG, McGuire BM. Influence of hepatic encephalopathy on health-related quality of life in patients with cirrhosis. Dig Dis Sci 2003;48:1622-6.

4. Younossi ZM, Boparai N, Price LL, et al. Health-related quality of life in chronic liver disease: the impact of type and severity of disease. Am J Gastroenterol 2001;96:2199-205.

5. Tsochatzis EA, Bosch J, Burroughs AK. New therapeutic paradigm for patients with cirrhosis. Hepatology 2012;56:1983-92.

6. Agrawal K, Kumar P, Markert R, et al. Risk factors for 30-day readmissions of individuals with decompensated cirrhosis. South Med J 2015;108:682-7.

7. Volk ML, Tocco RS, Bazick J, et al. Hospital readmissions among patients with decompensated cirrhosis. Am J Gastroenterol 2012;107:247-52.

8. Orem D. Nursing: concepts of practice. United States of America: McGraw-Hill Book Company, 1980:225.

9. Aish $A E$, Isenberg M. Effects of Orem-based nursing intervention on nutritional self-care of myocardial infarction patients. Int J Nurs Stud 1996;33:259-70.

10. Orem DE, Renpenning KM, Taylor SG. Self care theory in nursing: selected papers of Dorothea Orem. New York: Springer Pub, 2000:400.

11. Shutt JD, Robathan J, Vyas SK. Impact of a clinical nurse specialist on the treatment of chronic hepatitis C. Br J Nurs 2008;17:572-5.

12. Wigg AJ, McCormick R, Wundke R, et al. Efficacy of a chronic disease management model for patients with chronic liver failure. Clin Gastroenterol Hepatol 2013;11:850-8.

13. Biddle ML, Adler NR, Heath M, et al. Nurse-led clinic: effective and efficient delivery of assessment and review of patients with hepatitis B and C. Intern Med J 2014;44:581-5.

14. Grogan A, Timmins F. Side effects of treatment in patients with hepatitis C - implications for nurse specialist practice. Aust $J$ Adv Nurs 2009;27:8.

15. Schadewaldt V, Schultz T. Nurse-led clinics as an effective service for cardiac patients: results from a systematic review. Int $J$ Evid Based Healthc 2011;9:199-214.

16. Campbell NC, Thain J, Deans HG, et al. Secondary prevention clinics for coronary heart disease: randomised trial of effect on health. BMJ 1998;316:1434-7.

17. Murchie P, Campbell NC, Ritchie LD, et al. Effects of secondary prevention clinics on health status in patients with coronary heart disease: 4 year follow-up of a randomized trial in primary care. Fam Pract 2004;21:567-74. 
18. Chan AW, Tetzlaff JM, Altman DG, et al. SPIRIT 2013 statement: defining standard protocol items for clinical trials. Ann Intern Med 2013;158:200-7.

19. Hoffmann TC, Glasziou PP, Boutron I, et al. Better reporting of interventions: template for intervention description and replication (TIDieR) checklist and guide. BMJ 2014;348:g1687.

20. Miller W, Rollnick S, Interviewing M. Helping People Change. 3rd Edition. United states of America: The Guilford Press, 2013:482.

21. Hays RD, Sherbourne CD, Mazel RM. The RAND 36-Item Health Survey 1.0. Health Econ 1993;2:217-27.

22. Hays RD, Morales LS. The RAND-36 measure of health-related quality of life. Ann Med 2001;33:350-7.

23. Wilde B, Starrin B, Larsson G, et al. Quality of care from a patient perspective-a grounded theory studyScand. $J$ Caring Sci 1993; 7:113-20

24. Wilde Larsson B, Larsson G. Development of a short form of the quality from the patient's perspective (qpp) questionnaire. $J$ Clin Nurs 2002;11:681-7.

25. Pugh RN, Murray-Lyon IM, Dawson JL, et al. Transection of the oesophagus for bleeding oesophageal varices. Br J Surg 1973:60:646-9.

26. Durand F, Valla D. Assessment of prognosis of cirrhosis. Semin Liver Dis 2008;28:110-22.

27. Kamath PS, Wiesner RH, Malinchoc M, et al. A model to predict survival in patients with end-stage liver disease. Hepatology 2001;33:464-70.

28. Wiesner R, Edwards E, Freeman R, et al. Model for end-stage liver disease (MELD) and allocation of donor livers. Gastroenterology 2003;124:91-6.

29. Borhofen SM, Gerner C, Lehmann J, et al. The royal free hospital-nutritional prioritizing tool is an independent predictor of deterioration of liver function and survival in cirrhosis. Dig Dis Sci 2016;61:1735-43.

30. American Association for the Study of Liver DiseasesEuropean Association for the Study of the Liver. Hepatic encephalopathy in chronic liver disease: 2014 practice guideline by the European Association for the Study of the Liver and the American Association for the study of liver diseases. $J$ Hepatol 2014:61:642-59.

31. Bleibel W, Al-Osaimi AM. Hepatic encephalopathy. Saudi J Gastroenterol 2012;18:301-9.

32. Weissenborn K, Ennen JC, Schomerus H, et al. Neuropsychological characterization of hepatic encephalopathy. $J$ Hepatol 2001;34:768-73.

33. Elsass P, Christensen SE, Mortensen EL, et al. Discrimination between organic and hepatic encephalopathy by means of continuous reaction times. Liver 1985;5:29-34.

34. Sheridan SL, Halpern DJ, Viera AJ, et al. Interventions for individuals with low health literacy: a systematic review. $J$ Health Commun 2011;16 Suppl 3:30-54.

35. Weiss BD, Mays MZ, Martz W, et al. Quick assessment of literacy in primary care: the newest vital sign. Ann Fam Med 2005;3:514-22.

36. Laucis NC, Hays RD, Bhattacharyya T. Scoring the SF-36 in Orthopaedics: a brief guide. J Bone Joint Surg Am 2015;97:1628-34.

37. Team UR. UKATT Research Team. Effectiveness of treatment for alcohol problems: findings of the randomised UK alcohol treatment trial (UKATT). BMJ 2005;331:541

38. Samsa G, Edelman D, Rothman ML, et al. Determining clinically important differences in health status measures. Pharmacoeconomics 1999;15:141-55.

39. Julious SA. Sample sizes for clinical trials. United States of America: Chapman \& Hall/CRC, 2009:268.

40. Hotopf M. The pragmatic randomised controlled trial. Advances in Psychiatric Treatment 2002;8:326-33.

41. Moore GF, Audrey S, Barker M, et al. Process evaluation of complex interventions: medical research council guidance. BMJ 2015;350:h1258-7.

42. Les I, Doval E, Flavià M, et al. Quality of life in cirrhosis is related to potentially treatable factors. Eur J Gastroenterol Hepatol 2010;22:221-7. 